\title{
ORIENTACIÓN VOCACIONAL Y \\ PROFESIONAL EN COLEGIOS DE BAJO NIVEL \\ SOCIOECONÓMICO: PERCEPCIONES DE ORIENTADORES Y ESTUDIANTES
}

\section{Introducción}

Este artículo presenta los resultados de la investigación encargada por el Consejo Superior de Educación (CSE): "La orientación vocacionalprofesional en establecimientos de nivel socioeconómico bajo en el Gran Santiago: percepciones de orientadores y alumnos".

En los primeros capítulos se presenta la recolección de antecedentes, cuyas dos fuentes principales fueron los estudios y artículos disponibles en la materia y las conclusiones de expertos en orientación convocados especialmente para discutir los hallazgos de la revisión bibliográfica e identificar otros tópicos relevantes en el ejercicio de la labor orientadora.

El tercer capítulo resume la metodología de investigación escogida para analizar las percepciones de los orientadores y alumnos; se detalla la metodología de selección de la muestra y los criterios con los que se confeccionaron los instrumentos de recolección. El cuarto describe los principales hallazgos en los discursos de orientadores y alumnos.

Hacia el final se señalan las principales preguntas arrojadas por el estudio y se esbozan algunos criterios para el diseño de una política que refuerce los vínculos de sentido entre los sistemas de educación media y superior, a través de la labor de orientación vocacional-profesional. 


\section{Panorama sociohistórico de la educación en Chile}

El sistema de educación chilena ha sufrido importantes transformaciones en la casi totalidad de ámbitos de su funcionamiento desde la década de los ochenta en adelante. Durante esa década se transfiere la responsabilidad por la gestión del sistema escolar público a los gobiernos municipales. Asimismo, parte de los centros educacionales pasan a ser sostenidos por corporaciones privadas. En educación superior, en tanto, la presencia del Estado va progresivamente disminuyendo, las instituciones nacionales son desmembradas y las antiguas sedes regionales pasan a ser universidades derivadas. Junto con ello, se produce una proliferación de instituciones privadas en lo que ha sido caracterizado como "privatización y desregulación expansiva" (Brunner, 1990).

La dinámica expansiva de ambos sistemas constituye una característica de su funcionamiento durante la década de los ochenta, manteniéndose en los noventa con algunos cambios en sus orientaciones. En la educación media, por ejemplo, se aprecia un aumento de la cobertura, que ya a mediados de década llega a cifras superiores al 90\% del total de población en edad de cursar estudios secundarios. Se produce, además, un importante giro en el desarrollo curricular de los programas educacionales, desde la creación del programa de mejoramiento de la calidad y la equidad (MECE) hasta la instauración de la jornada escolar completa (JEC)․․ Las transformaciones también se reflejan en el paso de una definición estatal de planes y programas a un marco curricular de objetivos fundamentales y contenidos mínimos obligatorios que pretende otorgar libertad a los establecimientos para que elaboren sus propios planes y programas.

La educación superior, por su parte, intensifica su proceso hacia la masificación, en la medida en que se crean nuevos planteles y se consolidan los anteriores en el mencionado escenario expansivo. En lo

1 Proceso conjunto conocido como Reforma Educacional. 
que compete al Estado, toda nueva institución de educación superior debe seguir un proceso de reconocimiento oficial y de licenciamiento o supervisión de sus estructuras regulativas hasta que, llegado el momento, pueda demostrar capacidad de autorregulación. Además, se distinguen las carreras exclusivamente universitarias de aquellas profesionales y de los estudios técnicos.

El sistema educacional en su conjunto está cruzado por tendencias de desarrollo que afectan tanto a los estudiantes (en tanto individuos) como a las prácticas de funcionamiento interno de los centros educativos y la forma en que éstos se relacionan con el entorno social y laboral. La cobertura de educación media, por ejemplo, contribuye a proyectar un sistema de formación permanente, lo que sin duda ha tendido a modificar los imaginarios respecto de la educación; en efecto, éstos han ido desplazándose desde la preparación en ciertas competencias "para el resto de la vida" a una valoración de la formación "a lo largo de la vida" (Andaur, 2005).

Especialmente con relación al segmento de educación media científico-humanista, las oportunidades laborales de los egresados del sistema escolar se ven fuertemente condicionadas dependiendo de si han cursado o no estudios postsecundarios. Todo esto da cuenta de que se han producido importantes cambios en las relaciones entre sistema educativo y mercado laboral, que impactan y modifican las percepciones sociales respecto de la oferta formativa. Así, el vínculo de la educación superior respecto de su escenario productivo cada vez va distinguiendo más la formación profesional de la técnico/profesional como posibilidades distintas de trayectoria educativa.

La masividad constituye hoy una de las tendencias centrales de desarrollo de los sistemas de educación escolar y superior, transformando las condiciones en que se desarrolla el proceso formativo en el marco de un alargamiento general de la etapa juvenil del estudiante y de creciente retraso en la inserción laboral del mismo. También reordena las expectativas y, sobre la validación social que adquiere, la capacitación y las certificaciones de estudios 
especializados en la definición de la propia trayectoria formativa. Las motivaciones y condiciones de los estudiantes han cambiado entonces por la misma enseñanza obligatoria recibida, así como por el nuevo escenario que ofrece el sistema de educación superior.

Si en 1990 la cobertura total de la educación superior era de un 16\%, el año 2003 alcanzaba un 37,5\%. Para el conjunto socioeconómico estudiado, las cifras son elocuentes: "Si bien los principales incrementos se producen en los quintiles superiores, se triplica en todo el periodo la cobertura del $40 \%$ de los hogares de menores ingresos: de 4,4 al 14,5\% en Q1, de 7,8 a 21,3\% en Q2 y de 12,4 a 32,8 para Q3" (Cancino y Donoso, 2007).

Se debe hacer hincapié en que el mencionado carácter de masividad de la educación postsecundaria ha sido impulsado y fomentado mediante el mercado. El sistema de educación superior se encuentra inmerso en una dinámica expansiva en la que tanto las instituciones públicas como privadas (en grados y con énfasis distintos) deben competir para asegurar niveles razonables de matrícula nueva todos los años, destinando grandes recursos para acceder a los mismos establecimientos y exponer su oferta. Últimamente, se ha diagnosticado una segunda ola de expansión de sedes dependientes de instituciones de educación superior consolidadas en el escenario educativo (CNAP, 2003).

Otro aspecto relevante es la forma como la participación de los privados a la provisión de educación afecta las conductas institucionales e individuales, ya sea que se quiera resaltar positivamente la diversidad de la oferta entre la que los usuarios eligen hoy o la segmentación de calidad que supone la existencia de una gran cantidad de instituciones cuyo costo recae principalmente en la capacidad diferencial de pago o endeudamiento por parte de los estudiantes y sus familias.

Los imaginarios respecto de la oferta formativa (por parte de los postulantes) van siendo lentamente modificados, tal vez a un ritmo más pausado que la oferta misma, lo que deja en evidencia 
algunas de sus principales distorsiones. Las altas tasas de deserción en la educación superior reflejan en parte la relevancia del asunto (CSE, 2007).

En resumen, la educación media y la superior han transitado por procesos de modernización y masificación, evidenciándose que cada uno lo ha hecho de acuerdo con su propia lógica, inserto en un contexto de creciente valoración de la formación continua y de nivel superior por parte de la población ${ }^{2}$. Se puede decir, por esto, que entre estos sistemas se evidencia un proceso de desacople, esto es, que sus relaciones y vasos comunicantes hoy se encuentran poco clarificados, respondiendo más a la lógica interna de cada nivel educativo que a la racionalización de los vínculos que dan sentido a su continuidad en una trayectoria formativa concreta.

En particular, la literatura revisada se refiere a la hipótesis del desacople entre las expectativas generadas por la oferta formativa de nivel terciario, enfatizando el diagnóstico respecto de las capacidades para integrar, sistematizar y distribuir información relevante para la toma de decisiones por parte de los estudiantes en condiciones de seguir estudios postsecundarios, sobre todo si se piensa que gran parte de la información circulante es promoción comercial proveniente de las mismas instituciones de educación superior. Se trata de un tópico fundamental en la definición de la gama de opciones que posee el estudiante en el momento en que trabaja sobre su decisión de continuar estudios. En este contexto, la política pública no ha sido certera en dar con agentes capacitados para servir de mediadores entre ambos espacios educativos, lo que contribuye a acrecentar los límites de ambos para aumentar, ajustar y concretizar la información circulante sobre la oferta formativa hacia los principales demandantes en estas materias: los estudiantes.

2 Ver CIDE, "Encuesta nacional de actores del sistema educativo", años 2001 y 2003. 


\section{Los orientadores en el actual escenario educativo}

Con el fin de tener una panorámica respecto de las características de la labor orientadora, es necesario traer a la vista el conjunto de factores que condicionan su labor concreta, sus actividades y las relaciones de sentido que se instalan hoy en la educación media respecto de la educación superior. Por ello, la investigación se orientó inicialmente a recoger los elementos marco que caracterizan la función de los orientadores en el actual escenario chileno. Para esto se recurrió a la recolección bibliográfica. Los resultados de este "barrido" a través de la literatura existente fueron discutidos en mesas de trabajo con expertos en orientación, quienes añadieron nuevas aristas, modificaron otras y permitieron ponderar la importancia de las dimensiones que el tema posee. A continuación, se presenta una síntesis de las principales conclusiones de esta etapa.

\section{Función del orientador}

La orientación es definida como una parte consustancial del proceso educativo, que consiste en un apoyo específico al desarrollo personal de los estudiantes en el marco de los objetivos generales de la educación en sus niveles primario y secundario. Así, el proceso de orientación en las unidades educativas constituye "uno de los medios para lograr los fines de la educación, que tiene su esencia en la formación humana integral del joven como persona, concebida como ayuda permanente, para que los adolescentes sean capaces de plantear un proyecto de vida personal y para resolver situaciones, problemas y expectativas de distintos tipos: de la vida familiar, ciudadana, educacional, vocacional y profesional que la sociedad contemporánea le plantee" (De la Fuente y Núñez, 2002).

En lo que respecta a la esfera vocacional-profesional, proponemos entender el objetivo de la orientación como: lograr que los estudiantes tomen decisiones pertinentes y racionales según sus expectativas y necesidades, que estén informados y que tengan una visión amplia de los costos, las implicancias y los posibles resultados de tales decisiones. 
Los orientadores de establecimientos de enseñanza media son, potencialmente, agentes centrales en los establecimientos en la clarificación de oferta para la continuación de la formación de los estudiantes, revirtiendo o aminorando la brecha entre los sistemas de educación media y superior. En el orientador recae la tarea de generar procesos de elaboración de sentido para la definición de las trayectorias formativas de los alumnos, que sean capaces de contrarrestar la tendencia hacia la asimilación pasiva de expectativas del medio así como a la información de mercado, las que no necesariamente se ajustan a su realidad. Entonces, el orientador es el profesional capacitado para guiar dicho proceso, aunque hay acuerdo en que se trata de una responsabilidad que, finalmente, compete a toda la comunidad educativa.

La orientación vocacional-profesional se entenderá entonces como el proceso, planificado y coordinado técnicamente por el orientador, que tiene como principal objetivo acompañar, informar y asesorar a los estudiantes en la toma de decisión referida a la continuación de estudios, con perspectiva en su inserción laboral. El rol del orientador debiera estar enfocado en poner a disposición del estudiante las herramientas necesarias para que su decisión optimice la relación entre sus posibilidades, necesidades y expectativas, y a su vez sea coherente con la trayectoria biográfica trazada por él mismo.

\section{El contexto normativo}

La primera característica que resalta en la labor orientadora actual, es la falta de una normativa concreta que regule su marco de acción. En rigor, el único documento que permitía tener un grado mayor de claridad sobre la normativa que regula su función era el Decreto 600 del MINEDUC, derogado sin que haya sido reemplazado por un código similar.

Esto sin duda repercute en que su rol termine careciendo de especificidad, lo que provoca un efecto de dispersión de sus 
labores. El orientador debe realizar un gran número de funciones que no le corresponden, como labores administrativas o tareas que el resto del personal docente no realiza. El orientador lleva a cabo, además, las tareas que por formación se supone debiera cumplir en el establecimiento, en una gama que va desde actividades propias de la labor del psicólogo o del trabajador social, lo que merma sus posibilidades de éxito en los objetivos que se propone, entre ellos, en el plano vocacional.

\section{Competencias y formación}

En Chile, hasta 2002, en todos los establecimientos de más de 500 alumnos se contaba con orientadores o encargados de orientación. Entre ellos, el 88\% tenía formación específica y la población de orientadores se componía en un $66 \%$ por mujeres y en un $61 \%$ por personas mayores a 45 años (PIIE, 2002).

En la asamblea general de la Asociación Internacional para la Orientación Educacional y Profesional (AIOEP), realizada en Berna en 2003, se identificaron 10 competencias indispensables para el buen ejercicio de la acción orientadora, en el marco de las transformaciones que ha sufrido la educación inicial en el mundo. De esas competencias, un estudio encargado por Chilecalifica (Márquez et al., 2005) concluye que los programas académicos chilenos destinados a la formación de orientadores abarcan centralmente consejería, orientación educacional y consulta y coordinación.

De las siete restantes, cuatro de ellas (desarrollo de la carrera, gestión de la información, desarrollo grupal y comunitario y desarrollo del empleo) están por lo general ausentes, y tres (diagnóstico, investigación, y evaluación y gestión de programas y servicios) se presentan de forma muy marginal y sólo en algunos programas de postítulo o magíster, siendo justamente las competencias ausentes las que permitirían el contacto de la labor de orientación con la sociedad en dos de los campos más relevantes para sus objetivos: educación superior y mundo laboral. 
Otro de los estudios consultados señala que los orientadores cuentan con capacidad de generar vínculos, o bien aprovechar los existentes, con organismos como redes de salud, de prevención de drogas, de políticas sociales focalizadas, entre otros, pero: "(cuentan con) casi nulos contactos con el mundo laboral, empresas, asociaciones de empresarios, $\mathrm{OMIL}^{3} \mathrm{y}$ otros organismos que proveen información en materia laboral-profesional. (...) Los desafíos del mercado de trabajo moderno, en particular la necesidad de desarrollar la capacidad de emprender y de prepararse para cambiar el rumbo muchas veces durante la vida de trabajo, son temas que no aparecen" (Rossetti, 2003).

En el contexto de la masificación mediante el mercado que caracteriza el proceso de modernización educacional en Chile, estas carencias impactan directa o indirectamente en los mecanismos de que disponen los establecimientos de enseñanza media en general, y los orientadores en particular, respecto de la oferta de programas en educación superior, como charlas de instituciones, ferias, exposiciones, por ejemplo, la que en gran parte es información promocional de las mismas instituciones.

Por otro lado, la orientación entendida como una labor de apoyo al proceso educativo no se ejerce independientemente de las otras actividades en el interior de un establecimiento. Por ello, se espera que la orientación no sólo esté presente en el resto de las actividades formativas (por ejemplo, a partir de los objetivos fundamentales transversales), sino que la organización de diversas estructuras de trabajo entre los agentes educativos posibilita abrir canales para facilitar el impacto de la orientación en la globalidad del proceso. Sin embargo, a propósito del artículo del PIIE, Rafael Andaur comenta que: "si bien se establece que la labor de orientación debe ser una función compartida con todos los integrantes del liceo, el orientador tiende a concentrar su trabajo en la atención directa a los alumnos, y su trabajo en términos de orientación vocacional y

3 Oficina Municipal de Inserción Laboral. 
laboral está enfocado a promover el término de la escolaridad y la continuación de estudios, de preferencia universitarios, sin mayor referencia a las condiciones requeridas para llevar a cabo el proyecto y el contexto en el cual deberá desempeñarse" (Andaur, 2005).

El hecho de privilegiar la atención directa en escenarios donde la proporción entre orientadores y alumnos y es de uno a 600 o más, impacta en que un $74 \%$ de los estudiantes no conoce al orientador y un $78 \%$ nunca ha conversado con él (PIIE, 2002). Pero también redunda en pocas instancias para poder definir estrategias comunes entre profesores de aula, jefes de Unidad Técnico Pedagógica (UTP) y profesores jefes, y en la posibilidad de instalar en otros ámbitos de la educación la orientación vocacional-profesional.

\section{Orientación e información sobre educación superior}

En un estudio de CIEPLAN se identificaron distintas formas de segmentación en el acceso y uso de la información sobre educación superior, las que impactan en la toma de decisiones de los postulantes. Las que aparecieron como las más relevantes fueron: el nivel socioeconómico (NSE) de la familia, el puntaje en la Prueba de Aptitud Académica (PAA, actual PSU) del propio estudiante, el tipo de enseñanza que se brinda en su establecimiento (técnico-profesional o científico-humanista) y si el postulante es recién egresado de enseñanza media o no (Raczynski y Canales, 2000).

Además de las dimensiones que pueden considerarse estructurales (como las anteriores), el desigual acceso se ve potenciado por algunos aspectos de la cultura de la educación superior chilena que prima en ciertos establecimientos educacionales secundarios. Por ejemplo, hay colegios donde se evidencian barreras para que los jóvenes conozcan toda la gama de opciones disponibles, ya sea por vínculos directos entre colegios e instituciones de educación superior o porque la disponibilidad de dicha información podría implicar al establecimiento una importante fuga de matrícula. Asimismo, la "cultura de prestigio" de ciertos establecimientos secundarios (como los colegios particulares o muchos de los denominados "liceos 
emblemáticos") a menudo pasa por alto la información respecto de la enseñanza técnico-profesional.

Los estudiantes esperan y demandan que el establecimiento educacional actúe como fuente intermediadora de información y, especialmente, que tienda a buscar soluciones a las necesidades que no pueden ser abordadas de manera satisfactoria por las redes sociales familiares. Esta expectativa es particularmente clara en el segmento poblacional con menor capital económico, cultural y social; la mayoría de las veces se hace demanda concreta y se dirige al orientador mismo. Presentados los antecedentes anteriores sobre cómo se prioriza y organiza el trabajo de orientación vocacional-profesional, resulta comprensible que la función de canales de información de los establecimientos sea generalmente mal evaluada (Raczynski y Canales, 2000).

Las expectativas de los alumnos y sus familias están enfocadas, por lo general, hacia carreras de educación superior tradicionales, incluso cuando se percibe crecientemente que, en la actualidad, ellas ya no garantizan del mismo modo el acceso al trabajo. Por ello se señala que "a pesar de su genuino interés (de los orientadores) en que los jóvenes continúen estudios, son pocas las referencias al vasto universo de centros de formación técnica (CFT), institutos profesionales (IP), predominando para ello la asociación educación superior = universidad" (Raczynski y Canales, 2000).

Existen entonces en el imaginario social algunas representaciones fuertemente instaladas que enfatizan el valor de las carreras profesionales y se transforman en demandas concretas hacia el establecimiento. Se asume que la obtención de una profesión trae como efecto movilidad social ascendente, aminorando la atención respecto de otras variables que también podrían facilitar dicha movilidad. Estos imaginarios son consecuencia de la propia dinámica que ha asumido la estratificación de la sociedad chilena en el pasado, y se ven reforzados por la carencia de medios efectivos para desmitificarlos y proveer de una observación más pertinente en el actual contexto. 
En el caso de la orientación vocacional-profesional, son justamente los orientadores los llamados a entregar las herramientas a los estudiantes para que puedan analizar su contexto y no tomar sus decisiones sobre la base de imaginarios y representaciones que no necesariamente encuentran fundamentos en su situación actual. En la mayor parte de la literatura revisada el manejo y acceso a la información actualizada y pertinente es evaluada como un aspecto central para la toma de decisiones de los estudiantes.

Para los alumnos provenientes de estratos socioeconómicos bajos esta labor es doblemente importante, pues en el orientador recae la tarea de hacer visible una estructura de oportunidades que tenga en cuenta la correspondencia entre el perfil sociocultural de los alumnos y la valoración de las opciones que "circulan", así como la necesidad de trabajar sobre la misma segmentación de los sistemas de información existentes hoy para estos fines.

\section{Metodología de estudio}

A partir del escenario presentado, resulta relevante indagar en las percepciones construidas en establecimientos educacionales de nivel socioeconómico bajo respecto de la orientación vocacional en Chile. Primero, por la carencia de estudios que sistematicen las visiones e inquietudes que poseen los actores directa y cotidianamente involucrados en las relaciones de orientación vocacional (orientadores y alumnos), pero, además, por el aún incipiente desarrollo de líneas de investigación que profundicen en el perfil de los estudiantes en el nuevo contexto educacional.

La presente investigación fue enfocada hacia la caracterización de esas percepciones, ya que en estos establecimientos se conjugan los problemas de composición y organización del trabajo de orientación con el escaso capital social y cultural de los alumnos y su entorno. Además, el aumento de la matrícula en educación superior es impulsado significativamente por estudiantes provenientes de establecimientos municipales y subvencionados (CSE, 2007). La muestra de orientadores 
y alumnos para ser entrevistados fue recogida entonces desde establecimientos de comunas de nivel socioeconómico bajo. Para definirlas, se utilizó la clasificación de proporciones poblacionales por comuna de ADIMARK (2004), seleccionando las comunas de la Región Metropolitana que sumaban más proporción de población clasificada en los estratos C3, D y E, a saber: La Pintana (95,2\%), Cerro Navia $(93,6 \%)$, Lo Espejo $(92,8 \%)$ y Renca $(90,8 \%)$.

Se tomó una muestra al azar de ocho establecimientos pertenecientes a las cuatro comunas señaladas (un humanistacientífico y un técnico-profesional por cada comuna) y se citaron entrevistas con sus orientadores. Además, se organizaron dos grupos focales (divididos por la dependencia de los colegios, municipales y particular-subvencionados) para una muestra de alumnos de cada colegio. Entonces, se realizaron en total ocho entrevistas en profundidad con orientadores y dos focus groups con alumnos.

Tanto las entrevistas individuales como las grupales se realizaron según pautas semiestructuradas. Este carácter del instrumento permitió la emergencia de tópicos no considerados en la recolección de antecedentes.

En el proceso de análisis de la información se aplicaron dos codificaciones: una cerrada, orientada a clasificar la información obtenida en las entrevistas y grupos focales en las dimensiones definidas con anterioridad a la realización del terreno, que fueron justamente las dimensiones utilizadas para diseñar las pautas de las entrevistas y de los focus groups, y una segunda codificación con elementos de análisis de la teoría fundada, la que, a través del método comparativo constante, permite codificar abiertamente tanto de forma axial como selectiva.

La codificación cerrada permitió identificar nuevas categorías y códigos que emergieron de la información obtenida en los focus groups y entrevistas; la axial permitió identificar los fenómenos principales a través de la relación de códigos, mientras que la selectiva permitió la identificación de una categoría principal: el núcleo del fenómeno 
investigado. Los principales hallazgos de este estudio se presentan a continuación.

\section{Resultados del estudio}

Un punto de partida para exponer los principales resultados que arrojó el análisis de los discursos, tiene que ver con la claridad con que aparecen las múltiples referencias al perfil de los alumnos con que trabajan los orientadores: estos coinciden en definirlo en función de su situación de pobreza y vulnerabilidad social. Esta caracterización no sólo se refiere a los estudiantes en tanto individuos, sino que abarca una amplia red de relaciones que incluye a las familias y el entorno donde viven.

La condición de vulnerabilidad tiene componentes económicos (escasez de recursos), sociales (contextos familiar y social, grupos de referencia y grupo de pares) y culturales (referidos fundamentalmente a las aspiraciones y expectativas puestas en juego para organizar sus propias trayectorias biográficas), que influirían fuertemente en la labor de orientación:

"Acá el nivel socioeconómico y cultural es bajo, entonces ahí el niño viene, el Liceo a veces es el primer eslabón que tienen para un futuro de vida mejor, porque son poco motivados en la casa, carentes de esa parte, muchos niños en el Liceo que me imagino que hasta el fin de semana se quedan con lo que comen en el Liceo y el fin de semana no sé poh, porque son niños muy deprivados en lo socioeconómico, cultural y afectivo es una, son muy deprivados, entonces ese es el mayor obstáculo que nosotros encontramos, la deprivación de estos niños en esa parte, de las familias muy deprivados, no el cien por ciento, pero gran parte de ellos" (orientador, $\mathrm{M} / \mathrm{TP}^{4}$ ).

$4 \mathrm{M} / \mathrm{TP}$ se refiere a liceo municipal técnico-profesional, M/HC municipal humanista-científico, PS/TP particular subvencionado técnico-profesional y PS/HC a particular subvencionado humanista-científico. El colegio señalado como HP/TC era un liceo polivalente. 
Profundizando en la caracterización del perfil general de los estudiantes de estos establecimientos, podemos señalar que tal situación de vulnerabilidad se traduce en distintos tipos de problemas de los cuales el establecimiento y, en particular, el orientador deben hacerse cargo: problemas de aprendizaje y conductuales, carencias afectivas derivadas de la situación familiar y propensión a la deserción escolar.

\section{El vacío normativo como dimensión problemática.}

a. Enfoques respecto a la orientación (orientación educacional y orientación vocacional)

El análisis de esta dimensión identifica la manera en que los orientadores comentan los aspectos involucrados en su labor, dando cuenta también de sus percepciones acerca de la importancia relativa de estos aspectos en el contexto en que desarrollan el trabajo de orientación.

En lo que atañe a la orientación educacional, tiende a haber consenso en que se trata de un tipo de ayuda o apoyo que aborda múltiples temáticas que se dan en el marco del proceso educativo: académicas, emocionales, afectivas, familiares, personales, incluyendo la temática vocacional. Esta definición construida por los propios orientadores se plantea como una respuesta lógica a los principales problemas que deben encarar...

"es una disciplina que se encarga de entregar la herramienta de trabajo, tanto a alumnos como docentes, para resolver situaciones en el área académica, emocional y afectiva de los alumnos, fundamentalmente. Junto a eso (...) hacer un trabajo también en todo lo que tiene que ver con el proceso de decisión vocacional de los alumnos de enseñanza media, sobre todos en los alumnos de cuarto medio" (orientador, PS/HC/TP).

Profundizando en este tópico, se identifica un matiz de diferencia, en tanto algunos orientadores apuntan a un objetivo más 
general y abstracto (desarrollo integral del estudiante), mientras que otros se preocupan por entregar soluciones a problemas que se van dando a diario (apoyo en dificultades específicas).

"Son todas aquellas acciones pedagógicas y de apoyo o ayuda, destinadas al desarrollo integral y a la formación de los alumnos, tales como desarrollo de habilidades psicosociales, adquisición de valores, definición de proyecto de vida, manejo de información de servicios sociales y redes, entre otros" (orientador, PS/TP).

"Bueno la orientación es cuando uno orienta, ayuda y apoya al niño en cualquier tipo de dificultades o problemáticas que presente el niño, ya sea en la parte emocional (...) ahí se ven muchos casos de niños con problemas de rendimiento, emocionales, distintas áreas de problemáticas que puede tener el niño en cuanto a orientación (...) en drogas. Una serie de dificultades que puede presentar el niño ahí está la orientadora, como apoyo, una ayuda y orientación encauzándolas hacia donde podríamos ver la solución al problema" (orientador, M/TP).

Por otra parte, la orientación vocacional-profesional es percibida como un aspecto mucho más focalizado, en el sentido de que se acota en la identificación de las habilidades, intereses y vocación de los estudiantes, y entrega de información y apoyo en los procesos de decisión vocacional-profesional. En el fondo, se vincula con el apoyo que se le presta al estudiante en la construcción y proyección de su trayectoria formativa.

"es apoyar al alumno en la toma de decisiones, darle todas las herramientas para que él pueda optar o decidir su futuro estudiantil o laboral" (orientador, M- TP/HC).

Un aspecto de destacar es que, en el caso de los establecimientos educacionales técnico-profesionales, la orientación vocacional va asociada al proceso de elección de especialidad al ingresar a tercero medio. 
"Es como una ayuda, un apoyo, una línea de seguimiento del alumno en cuanto a sus habilidades, sus intereses y su vocación misma para que elijan una especialidad de acuerdo a estos intereses, habilidades y vocación de ellos mismos" (orientador, M/TP)

b. Orientación y misión de los establecimientos

La institucionalidad de un establecimiento escolar define en gran parte los principios de su labor educativa y la política explícita, que se traduce en el proyecto educativo, la misión y los objetivos de la enseñanza. Estos contribuyen con materializar y proyectar las percepciones, las valoraciones y el sentido de la educación que se entrega. El paso desde la misión a los objetivos es mediado por las percepciones y valoraciones hacia distintos aspectos del sistema educativo, las que no son específicas de cada establecimiento sino que corresponden a imaginarios sociales sobre los roles de la educación.

La misión institucional de un colegio define entonces el horizonte hacia donde apunta el proyecto educativo; éste supone una lectura e interpretación acerca de las condiciones en que se lleva a cabo la labor educativa (contexto económico, social, cultural, por ejemplo), el perfil de los estudiantes que ingresan al establecimiento y otros aspectos relevantes. La misión y los objetivos de los establecimientos también dan forma a los enfoques, las temáticas que se desarrollan y hacia dónde apuntan las tareas del orientador.

Tanto los orientadores como los estudiantes de colegios técnicoprofesionales subscriben la idea de que esta modalidad de la educación media se constituye en la mejor alternativa para estudiantes de nivel socioeconómico bajo, por cuanto entrega herramientas que facilitan su rápida inserción laboral. Se trata de un imaginario muy instalado en los mismos estudiantes de establecimientos técnico-profesionales, quienes valoran en sus discursos este tipo de enseñanza por entregarles mayores alternativas a la hora del egreso. 
La educación media científico-humanista, por su parte, es vista en general como una suerte de espacio intermedio en medio del tránsito hacia la enseñanza superior. Se plantea que para los estudiantes de nivel socioeconómico bajo este tipo de educación no es una buena alternativa, en la medida en que las posibilidades de continuar estudios postsecundarios son pocas, según reconocen ellos mismos. Los discursos, tanto de orientadores como de estudiantes, aclaran que la educación media técnico-profesional apunta a objetivos distintos que los de la educación científico-humanista, prepara a sus estudiantes sobre la base de objetivos distintos, los que pueden ser clasificados en las opciones "preparar para insertarse en mundo laboral" y "preparar para la continuidad de estudios en la educación superior".

"nuestra labor es prepararlos para el mundo del trabajo, diríamos, para un título técnico, de mando medio, esa es nuestra labor, pero, sin embargo, también nosotros los preparamos para que ellos también puedan ingresar a la educación superior, pero no como en un liceo científico humanista diríamos" (orientador, PS/TP).

"Aquí nosotros, suponte, nuestra misión dice que los alumnos puedan ingresar a educación superior, pero la educación superior para nosotros son las universidades, los institutos, los centros de formación técnica, las ramas de las Fuerzas Armadas ino es cierto? Entregarle todo el abanico de posibilidades que existe" (orientador, $\mathrm{M} / \mathrm{HC})$.

Ambos objetivos constituyen tipos puros que en la realidad de los liceos (humanista-científico y técnico-profesional) no se dan de manera tan diferenciada. La diversidad de problemas con que se trabaja en estos establecimientos tiene el efecto de reducir o ajustar estos objetivos. Por ejemplo, algunos establecimientos, más que buscar una real inserción laboral o una continuidad de estudios superiores, ponen la totalidad de sus esfuerzos en mantener a los alumnos dentro del sistema educativo, aspirando a que finalicen la enseñanza media. 
"retenerlos en el tema escolar los cuatro años ya es un desafío. Entonces, desde mi área y los profesores jefes, porque yo trabajo con los profesores jefes, estamos mucho más dedicados a... a eso, a que por lo menos terminen la enseñanza media" (orientadora, PS/TP).

Por esto, los objetivos que se proponen los colegios determinan el rango de acción de la orientación vocacional-profesional. Un ejemplo de lo anterior lo constituyen los establecimientos técnicoprofesionales, en los cuales la labor de orientación vocacional se enfoca en el apoyo en la elección de la especialidad y la búsqueda de las prácticas profesionales para los estudiantes, dando menos importancia al trabajo de información sobre oportunidades de continuidad de estudios.

Las relaciones entre el perfil de alumnos y el trabajo de orientación que aparecen en el discurso de los actores entrevistados son bastante elocuentes. Una de las características principales que se logró dimensionar en el análisis de las entrevistas se vincula con que, producto del tipo de estudiante y de su entorno social, éstos deben abocarse a una multiplicidad de temas que, en teoría, no debieran ser de su competencia. Esta situación es reconocida también por los mismos estudiantes.

"yo pienso que la orientadora es excelente, pero necesita otro orientador más porque para 1.300 alumnos que somos nosotras en el liceo, somos demasiados. Una que se encargue de los temas psicológicos, de los problemas socioeconómicos, de las vulneraciones que tienen los alumnos, una persona es demasiado. Ella es excelente, excelente persona, excelente orientadora, excelente psicóloga, eh no sé hasta matrona puede ser, pero necesita un apoyo, otra persona al lado" (estudiante, $\mathrm{M} / \mathrm{HC} / \mathrm{TC})$.

En términos generales, se puede plantear que, para el tipo de establecimientos educacionales estudiados, buena parte del trabajo 
que realizan los orientadores está relacionado con las temáticas y dificultades propias de trabajar con estudiantes que se encuentran en una situación de pobreza y vulnerabilidad social. A raíz de esta condición, surge una serie de problemas que demandan la atención y el trabajo del orientador: problemas de aprendizaje de los propios alumnos, situaciones conflictivas en las familias, traumas psicosociales asociados a carencias afectivas, drogadicción, alcoholismo, altas tasas de embarazos adolescentes, propensión a la deserción escolar, entre otros muchos.

"Pero también ocurre esto de apagar incendios de todo estilo, porque en un colegio como el nuestro ocurren cosas todos los días ¿Te fijas? Todos los días cosas en que hay que estar, de alguna manera, ayudando" (orientador, $\mathrm{M} / \mathrm{TP})$.

De este modo, el contexto de trabajo mismo va relegando a un segundo plano la labor de orientación vocacional-profesional.

"En el liceo mi labor es de, en cuanto orientación, como apoyo, ayuda a todos los niños que tienen algún problema que la profesora me derive. La profesora jefe me deriva los casos, veo de qué tipo de caso se puede hablar, si es problema emocional, problema conductual, si es problema de rendimiento, entonces... casuística, lo primero que hago es casuística" (orientador, M/TP).

Entonces, el perfil del estudiante, enmarcado en la lectura sobre dicho perfil plasmada en la misión y los objetivos del colegio, condiciona directamente la labor de orientación. Los orientadores plantean que, en su quehacer diario, la falta de normativa respecto de su rol y sentido se ve omitida en la práctica por la enorme cantidad y la magnitud de los problemas a los que deben responder como consecuencia del perfil de estudiantes con que trabajan y a quienes deben atender. 


\section{Competencias y recursos para la labor de orientación}

a. Recursos humanos y materiales

Tomando en cuenta las diferencias entre tipos de dependencia de los establecimientos, se hizo evidente (comparando los discursos) que los orientadores de establecimientos particulares subvencionados indicaban ventajas o fortalezas en aspectos que en los distintos establecimientos municipalizados aparecían como debilidades $u$ obstáculos a su labor.

Las ventajas o fortalezas mencionadas para los establecimientos particulares subvencionados se relacionan, en primer término, con la presencia de recursos materiales y humanos para llevar a cabo su tarea. Se manifiesta satisfacción con los recursos de que se dispone, ya que existe conciencia de que no se trata de una situación generalizada:

"Yo diría que yo aquí estoy en la gloria, y estoy en la gloria ¿por qué? Porque, primero, yo cuento con todos los recursos que necesito (...) los alumnos aquí tienen toda la tecnología para hacer las cosas, no como en otros colegios que tú tienes que pagar para que te fotocopien una prueba, en otros colegios tienes que pagar para que te saquen un documento, los alumnos, aquí no, todo es gratis, aquí el alumno no tiene que venir con el computador, no tiene que venir con un motor, no tiene que venir con nada, o sea, tiene todo aquí, el alumno, guía de trabajo, gratis, prueba impresa, gratis, o sea, tiene todo aquel acceso a la información" (orientador, $\mathrm{PS} / \mathrm{TP})$.

En segundo lugar, se valora el permanente apoyo que reciben de agentes externos (corrientemente, las fundaciones dueñas de colegios) para reflexionar y discutir el proyecto y los objetivos que se han propuesto como unidad educativa. Esto se puede vincular directamente con la dimensión anterior relativa a la delimitación del rol del orientador, en tanto los agentes "externos" posibilitan una mayor diferenciación de funciones y tareas dentro del establecimiento 
educacional. Así, por ejemplo, en algunos casos cuentan con un departamento encargado de la capacitación de los profesores, por lo tanto, dicha responsabilidad no corresponde al orientador:

"Lo que pasa que acá el colegio es de una fundación, la Fundación (...) entonces hay un... hay una parte que es capacitación (para profesores) ¿ya? Capacitación está a cargo de eso, capacitación está a cargo de trabajar con los profesores, trabajo con los niños, está en constante monitoreo de todas las clases, de lo que se haga, está como encima de todo eso ¿ya? (...) Eh... hay varias como actividades que están en eso, digamos. Pero yo, más que nada, trabajo con los niños (PS/HC).

Esta situación contrasta especialmente con los colegios de dependencia municipal. Los mismos aspectos que en el primer caso son valorados como vehículos de la labor orientadora, ahora aparecen como aspectos obstaculizadores o limitantes. Es así como el principal obstáculo identificado por los orientadores, tanto de establecimientos municipalizados como algunos particulares-subvencionados, es la falta de recursos humanos que permitan conformar equipos de trabajo capaces de dar respuesta a la multiplicidad de demandas que se les presentan.

"Así que soy la única que trabaja acá, son treinta y tres cursos, ocho cuartos medios y... mil cuatrocientos alumnos, más dos segundos medios, que equivale a cuarto medio del vespertino que también los atiendo yo" (orientador, M/TP/HC).

A partir de este diagnóstico, el discurso de los orientadores atribuye gran importancia a la existencia de un "equipo de orientación”, dado el perfil del estudiante con que trabajan. Mientras tanto, los problemas se enfrentan con el recurso humano disponible: orientador, profesores, jefes de unidad técnica. 
"Mi idea es de que un departamento de orientación que funcionara, para este sistema, para estos niños, porque hay que hacer ayudas familiares también ¿Te fijas? Hay que hacer apoyos familiares muchas veces, debiera ser un conjunto de personas que tuvieran relación con la temática" (orientador, M/TP).

El otro gran obstaculizador que identifican el resto de los orientadores tiene que ver con la deficiente infraestructura con que cuentan para realizar un trabajo de calidad:

"Eh... bueno, una oficina, lo digo porque a veces hay muchas... yo por ejemplo no tengo una oficina, tengo como un... un cubículo, que está, digamos a vista y paciencia de todo el mundo, de repente eso como que no te da como la privacidad suficiente para poder conversar con los alumnos, bueno, internet, o sea, computador, internet, bibliografía yo creo que es impo... o sea, por ejemplo, ojalá hubiera una biblioteca, un lugar donde uno pudiera consultar libros y con... con información lo más moderna posible, eh... bueno materiales de oficina yo creo que también ojalá que constante información y ofertas académicas recientes, o sea, como estar al tanto, digamos, con toda la información académica..." (orientador, PS-HC/TP).

"Mira ahora tengo aquí esto porque se lo tengo que entregar a los colegas y dime... un computador y una impresora, una impresora para poder imprimir, ni teléfono, ni ninguna de esas cosas tengo, entonces imagínate yo estoy a una cuadra de inspectoría y a mí me llaman de los institutos, me llaman de todas partes, a cada rato, y yo estoy con apoderados, con alumnos, entonces, tengo que salir corriendo" (orientador, M/ HC/TP). 
b. Formación profesional y aspectos de la labor orientadora En el contexto que venimos analizando, el trabajo con estudiantes de este perfil requiere de ciertas habilidades y conocimientos que la mayoría no maneja: nociones de psicología del adolescente, de trabajo con jóvenes en situación de vulnerabilidad social y de mediación y resolución de conflictos. El dominio de estos conocimientos se considera fundamental ante los principales problemas que deben enfrentar en estos colegios.

Respecto de la formación profesional de los entrevistados, predomina una inicial en pedagogía, básica o media. Casi todos ejercieron durante un tiempo su profesión dictando clases en aula para luego dedicarse a la orientación. Sólo dos de los entrevistados no presentan dicha formación inicial: uno es psicólogo y el otro pedagogo social.

La casi totalidad de quienes se desempeñan en el cargo son formados en orientación a través de postítulos o magíster en educación con mención en orientación. Ellos evaluaron positivamente los cursos de especialización recibidos, considerando que les entregaron una base sólida para realizar su trabajo. Sin embargo, también reconocen que el oficio de orientador se va aprendiendo con la práctica.

"Yo creo que la universidad lo que te da es seguridad y te da el título, entonces tú te sientes capaz de trabajar como orientador, los demás confían en ti, pero tú además tienes que ir aprendiendo y... y hasta con lo que uno manejaba de antes" (orientador, M/HC/TP).

Con relación a las fortalezas que les entrega su formación en orientación, el discurso de aquellos con formación inicial en pedagogía se mantiene en una caracterización superficial, sin lograr identificar fortalezas específicas para la realización de su trabajo. Por otra parte, quienes presentan una formación distinta (en este caso en psicología y en pedagogía social) ponen énfasis en las herramientas propias de su disciplina. El orientador formado en psicología valora su mayor conocimiento respecto de temas tales como psicología del 
adolescente, manejo de herramientas para identificar las habilidades de los estudiantes, rasgos de su personalidad e intereses. De acuerdo con la opinión de este profesional, dichos conocimientos y herramientas le permiten realizar un análisis más integral del estudiante para, posteriormente, asesorarlo en el tema vocacional. Por su parte, el pedagogo social identifica como fortalezas de su formación fundamentalmente los conocimientos y el manejo de herramientas para trabajar con jóvenes en situación de vulnerabilidad social, considerando temáticas tales como manejo de redes, psicología del adolescente, mediación y resolución de conflictos, entre otros.

Cabe destacar que las capacidades mencionadas por parte de estos profesionales son precisamente aquellas que el resto de los orientadores identifican como debilidades de su formación, y en las cuales consideran necesario capacitarse a través de cursos y diplomados, o bien informarse a través de colegas que tengan un mayor manejo de estas temáticas:

"yo creo que nos falta más por ejemplo, cosas de manejos de cosas psicológicas, diríamos, porque ya el orientador de repente se transforma en un psicólogo" (orientador, $\mathrm{PS} / \mathrm{TP})$.

El dominio de estas temáticas (que los orientadores distinguen ya como fortalezas, ya como debilidades) adquiere relevancia en la medida en que son las principales demandas que deben enfrentar los orientadores de establecimientos educacionales que trabajan con estudiantes de nivel socioeconómico bajo y en situación de vulnerabilidad social:

"No estaba así como ahora, esa situación ahora la hemos recibido a través de los cursos de perfeccionamiento, todo esto de lo que es la mediación, por ejemplo, en el colegio nuestro tenemos este asunto de mediación, tenemos varios colegas que están haciendo estudio sobre mediación, hay como cuatro colegas de un par de cursos... quienes estuvieron todo el año pasado 
estudiando para poder ver cómo solucionar conflictos. ¿Te fijas? Mediación de conflictos. Entonces, como esos materiales igualmente llegan acá, uno tiene acceso, por último, aunque no haya hecho el curso, tiene acceso a poderlo leer" (orientador, M/TP).

Uno de los hallazgos del presente estudio surgió al momento de hablar de las debilidades de la formación de quienes ejercen la labor de orientación y se relaciona con el tema específicamente vocacional de los estudiantes. De acuerdo con sus discursos, los orientadores han podido suplir esta debilidad sólo a través de la práctica misma de su oficio.

"Porque no recuerdo como haber tenido... yo creo que después eso lo adquirí yo aquí en la práctica como trabajar lo más... lo vocacional" (orientador, M/HC).

"Pero quizás en términos de la cuestión más... metodológica, por ejemplo, para trabajar los temas vocacionales, o los temas de elección profesional, yo creo que más o menos, por ejemplo yo no... yo aprendí en el quehacer cuando tenía que buscar información" (orientador, PS/ TP).

A partir de la identificación de las fortalezas y debilidades de su formación, los orientadores construyen un perfil del ideal de las competencias requeridas para trabajar en contextos de pobreza y vulnerabilidad social. Dentro de este perfil se plantea como indispensable el manejo de las temáticas de convivencia y mediación y resolución de conflictos, ya que así lo exige el perfil del estudiante que recibe el tipo de establecimientos seleccionados para este estudio.

En el campo específico de la orientación vocacional-profesional, los entrevistados dan cuenta de tres temáticas fundamentales de apoyo: identificación de habilidades, intereses y construcción de un proyecto personal; entrega y manejo de información que facilite la toma de decisiones y, finalmente, ajuste de expectativas de los 
estudiantes a la realidad, cuestión esencial, considerando el contexto socioeconómico de éstos.

Este último tópico se ve relacionado con la proactividad y la capacidad de contextualizar las expectativas de futuro de los estudiantes. La mayoría de los orientadores reconoce en los alumnos cierta tendencia a hacer una lectura "irreal" de sus posibilidades una vez egresados de la enseñanza media, distorsionada, en gran medida, por el exceso de oferta publicitaria. Ello hace necesario un trabajo específico sobre las expectativas que ellos se plantean en función de sus habilidades y características personales.

"Yo creo que lo indispensable, como te decía, es ver... contextualizar... todo lo que quieren estos niños, contextualizarlo a su realidad, eso es elemental, o sea, si quiere estudiar en una universidad privada, ¿cuánto cuesta una universidad privada? Y contextualizar también sus notas, llevarlos a la realidad... si tienen un promedio cuatro y tanto... difícilmente les va a alcanzar para una universidad... o sea, es casi imposible que saquen una buena PSU, si no se preparan ni nada... alcanzar una universidad tradicional. Entonces, yo creo que, lo más importante, como orientador yo creo, es conocer a cada alumno, verle sus habilidades, sus... sus características de personalidad, su integridad como persona y ver sus preferencias y ahí orientarlo a qué posibilidades tiene, pero a posibilidades que sean accesible" (orientadora, $\mathrm{PS} / \mathrm{HC})$.

La escasez de recursos humanos y materiales, sumada a las contingencias (y los medios para resolverlas) que aparecen como problemas inmediatos, da como resultado el que sean pocas las horas destinadas a orientación vocacional-profesional. Esta escasez de horas no sólo debe al exceso de responsabilidades, sino que también tiene que ver con la mentada carencia de una normativa que establezca las horas destinadas para tal efecto: 
"Siempre se plantea el tema en el fondo de que uno está casi a la merced de la buena disposición o mala disposición del director. No existe, o sea de hecho, es de libre elección la cantidad de horas de orientación, ponte tú, que hay en la enseñanza media. Nosotros en tercero y cuarto tenemos una sola hora, un tiempo que no es suficiente para trabajar todos los temas que deberían ser considerados" (orientador, PS/HC/TP).

\section{Sentido de la orientación y expectativas de sus actores}

Esta dimensión permite abordar un conjunto de imaginarios sociales que, tanto orientadores como estudiantes, construyen desde su experiencia acerca de lo que se espera que la orientación logre. Para una mejor comprensión de estos imaginarios se procedió a distinguir dos temáticas acerca de las cuales éstos tratan (a y b).

a. Percepciones y expectativas con relación a la labor del orientador De acuerdo con la percepción de los propios orientadores, su labor debe centrarse en apoyar a los estudiantes; sin embargo reconocen que muchas veces se ven sobrepasados producto del exceso y la diversidad de demandas que deben abordar, lo que finalmente tiene como resultado un descuido de las áreas centrales.

"Cuando yo como orientador veo que el problema me sobrepasa, yo tengo que mandarlo o al psicólogo, o al neurólogo o al psiquiatra y de hecho tenemos chiquillos en psicólogo, en neurólogo y en psiquiatra ¿ya? Por lo tanto, uno sabe hasta donde puede llegar, y uno no puede abarcar otros campos que no le corresponden, diríamos, porque uno tampoco está preparado, o sea uno... uno puede saber algo, diríamos, pero yo creo que pastelero a sus pasteles no más poh, ¿me entiende?" (orientador, $\mathrm{PS} / \mathrm{TP})$. 
Respecto de aquello que los orientadores perciben como expectativas de los estudiantes, lo primero que resaltan es que existe desconocimiento sobre el trabajo del orientador.

"Al principio son reacios a ir con uno porque -señorita y por qué me va a llevar si yo no estoy loco- entonces porque tienen esa idea de que si van a hablar con uno es porque está loco o que está enfermo -ah! Estai enfermo-, le gritan los otros, pero después ven que uno no es para nada... sino que uno es solamente como de apoyo, ayuda, de mala gana van, pero después se dan cuenta y ven que... y después van donde uno -señorita, ¿y cuando me va a volver a llamar?- pero en general se dan cuenta que uno solamente está para ayudarlos, apoyarlos a ellos" (orientador, M/TP).

Una de las principales expectativas de los estudiantes de establecimientos científico-humanistas percibidas en estos discursos, es que el orientador realice la labor de guía, en el sentido de mostrarles las distintas alternativas por las que puede optar en términos de su futuro vocacional-profesional. En ese sentido, la entrega de información pertinente se transforma en una importante expectativa.

"Más que nada como información en cuanto a las carreras, información en cuanto a becas, a todo lo que... es como de apoyo a cuanto... a la decisión que ellos tomen, o sea, ser como guía a lo que ellos quieren" (orientador, PS/HC).

Tomando en consideración el perfil del estudiante con el cual trabajan, los orientadores sostienen que otra de las expectativas relevantes hacia su labor es que el profesional cuente con mayor cantidad de tiempo destinada a la atención de problemas individuales.

"Yo creo que ellos esperarían a lo mejor que yo tuviera más tiempo para poder atenderlos, digamos, y para poder atender de forma individual muchos casos... en el caso 
nuestro, yo diría que hay mucha demanda de atención de casos con niños con problemáticas individuales, que son muy dispares unas de otras" (orientador, M/TP).

En opinión de los orientadores, de ellos se espera que:

- Entreguen información sobre distintas opciones para la continuidad de estudios superiores u otras alternativas. La información requerida dependerá del tipo de especialidad del establecimiento educacional. Mientras en los establecimientos técnico-profesionales prima la demanda por información acerca de institutos profesionales, en los colegios científico-humanistas y polivalentes se pide información acerca de universidades, específicamente sobre sus criterios de selección y el campo laboral de las distintas carreras, además de datos sobre ayudas estudiantiles disponibles y condiciones para postular.

- Realicen los trámites y gestiones necesarias para que los estudiantes obtengan, o al menos postulen a, las becas disponibles.

- Coordinen visitas y charlas por parte de profesionales y empleadores.

- Dediquen un mayor tiempo para atender problemas de carácter personal de los estudiantes.

Por su parte, entre los mismos estudiantes hay coincidencia en que las principales demandas hacia el orientador se relacionan con la solicitud de información sobre continuidad de estudios superiores y con la atención individual de problemas personales. Además, desde el estamento directivo se demanda que el orientador realice algún tipo de trabajo con los profesores, de manera de instalar en ellos las capacidades y habilidades necesarias para hacerse cargo en forma autónoma de solicitudes generales de los alumnos en el aspecto vocacional-profesional.

Con relación a la información sobre la educación superior, una de las principales demandas se vincula con la calidad de la misma, característica que se identifica con el orientador actualizado. 
"Eso yo creo, la información como más actualizada de todas las cosas. Porque hay información, pero uno ve la fecha y de repente son de como dos o tres años atrás" (orientador PS/HC).

Los orientadores identifican temas que para ellos resultan más relevantes y que se relacionan con la lectura que hacen ellos mismos respecto de la información que necesitan y solicitan los estudiantes:

- Posibilidades de becas que se ajusten al perfil de estudiantes con que trabajan. Los orientadores señalan que, si bien perciben una oferta creciente de becas, no disponen de información que les permita identificar las posibilidades reales que sus alumnos tienen de acceder a ellas.

- Relación entre carreras y mercado laboral: características de la inserción (sueldos, tiempo en que la inserción se produce, entre otros).

- Educación técnico-profesional y mayor información relativa a institutos profesionales y centros de formación técnica: punto interesante, en la medida en que revela la importancia que tiene la formación de los mismos orientadores en la tarea que realizan.

- Contenidos específicos de las carreras: mallas, duración, énfasis, en qué se distinguen de similares carreras ofrecidas por otras instituciones, entre otros datos.

- Calidad de las instituciones de educación superior menos conocidas que aparecen en la gama de oferta para la continuación de estudios; la información sobre la calidad, establecen, debe ser fidedigna, confiable y actualizada.

Muchos de estos temas corresponden a qué demandan los mismos estudiantes: fundamentalmente, información más específica sobre arancel y malla curricular y acerca del campo laboral de las carreras. En ese sentido, hay una correspondencia en cuanto a las temáticas de información más relevantes. En efecto, se pudo percibir que los estudiantes son bastante críticos con relación a los datos que entregan las universidades, ya que los consideran demasiado generales. 
"Te dan la malla curricular pero así, no específica, entonces no te explican de qué se trata cada ramo, ni te explican que materia específicamente vai a ver, solamente explican la malla y así como qué ramos son, los nombres y te explican el arancel y eso. Entonces encuentro que la información que te dan de la universidad no me sirve tanto" (estudiante, PS/TP).

Respecto de las fuentes de información disponibles para los estudiantes, los orientadores reconocen que son variadas, poniendo énfasis en la importancia creciente de internet. Sin embargo, plantean que no basta con que la información se encuentre en la red, ya que su búsqueda dependerá de la motivación y el interés de los propios estudiantes.

Los estudiantes señalan que sus fuentes son internet, amigos, los propios profesores (en el caso de colegios técnico-profesionales, especialmente los jefes de especialidad) y las visitas a las instituciones de educación superior. Lo interesante es que, de acuerdo con lo que señalan los estudiantes, lo más relevante para mantenerse informado y tomar decisiones adecuadas para el futuro es el propio interés.

b. Percepciones y expectativas con relación al futuro de los estudiantes

Esta subdimensión recoge los imaginarios sociales que orientadores y estudiantes han construido respecto del futuro de estos últimos.

Los orientadores señalan a lo menos cuatro expectativas que tendrían los estudiantes acerca de su futuro inmediato:

- Culminación de la enseñanza media y obtención de su título técnico con el objetivo de insertarse laboralmente. Esta expectativa es enunciada con mayor claridad y convencimiento en el discurso de los orientadores de establecimientos técnico-profesionales.

- Ausencia de expectativas de educación superior. La continuidad de estudios en la educación superior no sería una expectativa general, o bien es percibida como imposible de acuerdo con su condición socioeconómica. 
- Continuación de estudios superiores. Esta expectativa es señalada generalmente (pero no con exclusividad) por orientadores de establecimientos científico-humanistas y polivalentes. Se contemplan las distintas posibilidades de educación superior; sin embargo, los estudiantes de establecimientos científicohumanistas tendrían como expectativa mayoritariamente los estudios universitarios.

- Otras expectativas más allá de la educación superior. Una de ellas se vincula con la realización del servicio militar y continuar en la Fuerzas Armadas.

Por otra parte, podemos situar las expectativas de carácter más general, asociadas a que los estudiantes tengan ambiciones de futuro y a que sean capaces de formarse un "proyecto de vida". Esta sería más bien una aspiración que no distingue ni la dependencia ni el tipo de enseñanza del establecimiento, y que se sustenta en la idea de que los propios estudiantes puedan romper el círculo de la pobreza y vulnerabilidad social en que se encuentran.

"Pero... que los chiquillos tomen decisiones es complicado acá, así que ojalá el cabro pudiera tomar una decisión de lo que quiere hacer de su vida, que ya es difícil, están sumamente determinados o influenciados por todo lo que les está pasando en la vida" (orientador, PS/TP).

Los estudiantes, por su parte, señalan que los profesores y directivos de los establecimientos esperan que ellos puedan cumplir sus metas y propuestas personales, que lleguen a ser "alguien en la vida" y, por otro lado, que puedan continuar estudios en la educación superior. Asimismo, las propias expectativas de estos estudiantes entrevistados remiten a sus deseos por continuar estudios superiores, ya sea en institutos profesionales o universidades. Sin embargo, cuando el discurso de los estudiantes ya no se refiere a ellos mismos sino a la generalidad de los estudiantes, la percepción acerca de las expectativas cambia. De ellos se espera que, como máximo, egresen 
de la enseñanza media ${ }^{5}$. Con esto reconocen que la realidad de gran parte de sus compañeros es distinta a la propia, sobre todo en el caso de los establecimientos educacionales municipalizados:

"El 95\% de mi curso por lo menos. En mi curso somos como cinco o seis los que estamos interesados realmente en entrar a la universidad. Los demás, ¡vayan a saber ellos lo que quieren! Pero quizás, si ellos supieran realmente, una carrera técnica, que cuesta mucho más barata que una universidad, quizás se pueden interesar más en estudiar algo técnico y no quedarse solamente con el cuarto medio, con la especialidad que estudiaron. Claro, sabemos que eso es solamente un principio, casi nada de lo que uno debiera saber. Y la posibilidad de una universidad la ven como muy lejana" (estudiante, $\mathrm{M} / \mathrm{TP})$.

Ellos mismos esbozan algunas explicaciones acerca de este alta tasa de alumnos para quienes la continuidad de estudios superiores no constituye una expectativa: su propia condición económica (la cual se ve relacionada con un bajo capital cultural), la necesidad de generar ingresos de manera rápida, el entorno como obstaculizador de expectativas (fundamentalmente la familia como factor central en la presencia o ausencia de éstas), entre otras razones.

En los discursos de los estudiantes es posible observar con claridad lo que se han propuesto para el futuro: son capaces de describir claramente sus objetivos, lo cual revela una planificación coherente de su proyecto de vida y su proyecto formativo. Todo esto es puesto de relieve al momento de definir sus expectativas.

5 Aún cuando se solicitó a los orientadores que contactaran a estudiantes con distintos proyectos una vez egresados de enseñanza media, finalmente los asistentes a los focus groups fueron principalmente los mejores alumnos de cada establecimiento, seguramente porque eran quienes tenían mayor disposición a participar y por las reticencias de los orientadores a contactar alumnos con mal desempeño escolar. 
"Entonces uno tiene que ir viendo más que nada lo que va pasando en el camino, pero sí es bueno planificarlo de antes. Hay que tener claro que van a haber adversidades con las cuales uno va a tener que ir luchando, por ejemplo, si trabajo va a haber tiempos en que no voy a poder estudiar porque tengo que terminar un trabajo, entonces también no es tanto como para planificarlo, sino tener claro lo que uno quiere hacer. Si yo quiero terminar la carrera, hacer lo que sea para terminarla" (estudiante, M/HC/TP).

Con relación al fundamento de la elección de una carrera profesional, se mencionan tres elementos: la vocación (a través de determinada profesión es posible ayudar a otras personas, como horizonte de realización personal), los gustos e intereses personales, y la búsqueda de un respaldo económico.

\section{Principales hallazgos y conclusiones}

La labor de los orientadores es, en general, evaluada positivamente por los estudiantes. Pero los propios alumnos ponen la voz de alerta cuando indican que su impacto sería mucho mayor si se hicieran cargo de menos temas o fueran apoyados por un equipo más amplio. Así, los estudiantes ponen la mirada en un aspecto que, a nuestro juicio, constituye la pregunta central para abordar el problema de la orientación en Chile: ¿cuáles son las condiciones necesarias para que los orientadores realicen de manera adecuada sus tareas?

Si asumimos como efectivo lo que nos cuentan los orientadores, debemos entender que, en medio de las múltiples dimensiones atendidas, éstos priorizan "en la práctica" sus tareas cotidianas, relegando a segundo plano el acompañamiento a la definición de la trayectoria formativa en alumnos con expectativas de continuar estudios. La multiplicidad de demandas, sumada a la escasez de recursos humanos y materiales con que cuentan los orientadores, hace difícil la clarificación de sus funciones: la multifuncionalidad atenta no sólo contra un buen proceso de apoyo a la orientación 
vocacional-profesional, sino que indica, en general, un llamado de atención respecto de la función específica que el establecimiento le asigna.

La orientación escolar constituye aún en muchos aspectos una labor que tiende hacia la integralidad de su vínculo con el proceso formativo, esto es, es percibida como un aspecto consustancial al proceso educativo mismo, y se le demanda en consecuencia. El hecho de que las expectativas sobre el orientador se conviertan con frecuencia en demandas concretas, limita las posibilidades de parcelar la labor orientadora para brindar apoyo externo a la misma. Dicho de otra manera, se debe considerar que la orientación educacional (como totalidad de la práctica de los orientadores) predomina sobre la orientación vocacional-profesional (aspecto específico de ésta), incluso allí donde esta última debiera ser prioritaria, como en los cursos avanzados de la educación media.

A lo anterior se añade el hecho de que las herramientas de trabajo para la dimensión vocacional-profesional son evaluadas como un "aprendizaje práctico" más que un área de formación en orientación. Se puede comprender, entonces, que los marcos de sentido para la organización de actividades y tareas del área vocacional pocas veces pueden ser desvinculadas de las urgencias del contexto. Tanto los requerimientos coyunturales del establecimiento, de los estudiantes y de su entorno (social, familiar, cultural), así como la propia disposición del orientador hacia sus tareas, parece conducir a la conclusión de que las proyecciones que se puedan hacer respecto de facilitar el acceso a algunas herramientas (que hoy resultan imprescindibles) deben ser integradas a la dinámica misma de la labor, al menos hasta que no se visualicen transformaciones importantes en la definición de funciones de la orientación en la educación secundaria.

La carencia de regulación normativa de las funciones, la multiplicidad de labores y las dificultades propias del trabajo en condiciones de pobreza y vulnerabilidad social impactan de manera importante en la efectividad de la orientación vocacional-profesional. 
Por esto, las tareas específicas que corresponden a este aspecto de la orientación se ven desplazadas a momentos puntuales del calendario escolar (inscripción en pruebas de selección, postulaciones, entre otros) y tal ordenamiento hace necesario, tanto en orientadores como en estudiantes, un discurso que justifique esta práctica en relación con los tres elementos ya mencionados: la falta de tiempo (indicativa de cierta soledad respecto de las expectativas puestas en el apoyo), la carencia de recursos y la homogeneización de procedimientos de evaluación vocacional (los que muchas veces dificultan, más que promueven, soluciones a las demandas de orientación vocacional).

Tales discursos justificatorios se asocian con una suerte de naturalización de esta condición, lo que conforma un imaginario construido desde la precariedad (curricular, material y humana) y que, además, pone como horizonte una "orientación ideal", sustentada menos en una interrogación y problematización acerca del sentido de la propia práctica y más en los síntomas evidentes del mal funcionamiento de la misma.

Mientras tanto, la educación superior continúa masificándose y constituyendo cada vez más un concreto punto de referencia para las trayectorias biográficas de los estudiantes de enseñanza media. Si la hipótesis del desacople puede alcanzar algún grado de explicación del fenómeno, resulta sugerente vincularla con el hecho de que ni los orientadores ni los estudiantes parecen dispuestos a reducir las alternativas de continuación de estudios a un problema de mercado. Pero esa suerte de resistencia frente a la oferta educativa publicitaria (como dijimos, prominentemente promocional) no encuentra canales sólidos para constituirse en apoyos a la proyección de la continuación de estudios, y más bien los actores insertos cotidianamente en el problema terminan por naturalizar tal vacío ante la escasez de recursos en los colegios y la abrumadora primacía de la oferta publicitaria. Queda claro, además, que los orientadores cuentan con escasas posibilidades para profundizar en un conocimiento (panorámico pero completo) respecto de las alternativas de estudios superiores. Si bien se enuncia claramente que esta tarea no es de exclusiva responsabilidad 
del orientador, sí recae en él en la práctica, y así el desconocimiento de las nuevas condiciones de la educación superior aparece como un límite importante para el apoyo vocacional.

Es indicativo que uno de los principales temas emergentes en los discursos de orientadores se relacione con que la definición específica de la labor vocacional de los orientadores de establecimientos técnicoprofesionales se confronte en menor medida con los problemas derivados de la situación de pobreza y vulnerabilidad social, si se compara con la orientación llevada a cabo en colegios científicohumanistas. En esto influye fuertemente la misión educativa del establecimiento, y para los colegios técnico-profesionales redunda en la percepción de que la indefinición generalizada del rol de la orientación en la educación media chilena tiene en ellos un menor impacto negativo. La orientación vocacional-profesional se torna un apoyo más concreto en la educación técnica de nivel medio que en la formación humanista-científica, pues cuenta con un marco más preciso de labores para orientar en este nivel: elección de especialidad, seguimiento y orientación a alumnos destacados respecto de continuación de estudios, planificación de charlas y apoyos en instituciones externas más vinculadas con el área de formación actual, señalan una claridad respecto del propio rol mucho menos presente en orientadores de colegios científico-humanistas.

Los problemas que emergen en esta labor difícilmente puedan ser abordados en forma aislada por la política pública que tome cartas en el asunto. En este plano, tampoco puede cubrir todas las necesidades que se han diagnosticado, pero sí mostrar la posibilidad de proyectar la conformación de alianzas estratégicas entre organismos capacitados para cubrir aspectos puntuales. Esto, sin duda, impactaría positivamente en la recepción y la valoración de la orientación, y tendería a incrementar su eficacia.

Lo dicho, no debe hacer perder de vista que, de todos modos, hace falta un rediseño general de la práctica orientadora que contemple y resuelva tópicos como los señalados (capacitación continua, 
disponibilidad de recursos materiales y humanos, familiarización con el contexto de la educación superior y sus aspectos específicos, normativas claras respecto de horas destinadas a estos propósitos) en el marco de una reelaboración global del sentido de la práctica orientadora, de sus principales objetivos y de los roles que debe cumplir en la educación secundaria. Sin duda, esto contribuiría a mejorar los vínculos de sentido entre los espacios educacionales secundario y superior, cruzados hoy por las dinámicas de masificación de la enseñanza en Chile.

\section{Referencias bibliográficas}

ADIMARK (2004) Mapa socioeconómico de Chile. (Sitio en Internet) Disponible en http://www.adimark.cl/noticias/index.act

Andaur, R. (2005) Nuevos rumbos para la orientación en Chile. (Sitio en Internet) Disponible en http://www.chilecalifica.cl/prc/n-0-nuevosrumbosovl. doc

Brunner, J. J. (1990) Educación superior en América Latina: cambios y desafios. Santiago de Chile: F.C.E.

Cancino, V. y Donoso, S. (2007) Caracterización socioeconómica de los estudiantes de educación superior. Calidad en la Educación, 26, pp. 205-244.

Comisión Nacional de Acreditación de Pregrado (2003) Sedes de instituciones de la educación superior en Chile. Documento de Trabajo CNAP. (Sitio en Internet) Disponible en http://www.cnap.cl/estypub/ informe\%20sedes\%20(2003).pdf

Consejo Superior de Educación (2007) Estadísticas sobre tasas de retención, INDICES (Sitio en Internet) Disponible en: http://www.cse.cl/public/ Secciones/seccionestadisticas/estadisticas_sistema_2006.aspx

De la Fuente, L. y Núñez, E. (2002) La orientación educacional en la enseñanza media municipalizada: Las representaciones sociales de la orientación en orientadores y profesores jefes de los liceos municipalizados de Maipú. Memoria para optar al título profesional de psicóloga. Santiago de Chile: Universidad de Chile.

Márquez, P.; Muñoz A. y Marcone R. (2005) Experiencias nacionales e internacionales, políticas e iniciativas de formación profesional de 
orientadores en el contexto de la relación de educación y trabajo. Santiago de Chile: II Seminario Internacional de Orientación Vocacional y Laboral: una nueva formación para orientadores.

Ministerio de Educación (2007): Indicadores de la educación en Chile, año 2005. (Sitio en Internet) Disponible en http://w3app.mineduc.cl/DedPublico/ indicadores_educacion

Programa Interdisciplinario de Investigaciones en Educación (2002) La orientación y el rol socio-profesional de los orientadores en los establecimientos educacionales chilenos. Santiago de Chile: PIIE.

Programa Interdisciplinario de Investigaciones en Educación (2004) Estudio de interés vocacional y laboral en jóvenes de enseñanza media. Resumen ejecutivo. Santiago de Chile: PIIE.

Raczynski, D. y Canales A. (2000). Demanda de Información sobre las carreras de pregrado en la educación superior chilena. Santiago de Chile: CIEPLAN.

Rossetti, Josefina (2003) Diagnóstico sobre la orientación y el rol socio-profesional de los orientadores en los establecimientos educacionales chilenos. Santiago de Chile: PIIE.

Unidad de Currículo y Evaluación del Programa de Mejoramiento de la Calidad y Equidad de la Educación, MECE (1997) Propuesta para el proceso de orientación en educación media. Santiago de Chile: Ministerio de Educación.

Recibido: 22 de octubre de 2007

Aceptado: 22 de mayo de 2008 